\title{
50-GHz Gain Switching and Period Doubling Using an Optical Injection Locked Cavity-enhanced DFB Laser
}

\author{
Zhixin Liu ${ }^{1}$, Yasuhiro Matsui ${ }^{2}$, Richard Schatz ${ }^{3}$, Ferdous Khan ${ }^{2}$, Martin Kwakernaak ${ }^{2}$, Tsurugi Sudo ${ }^{2}$ \\ ${ }^{I}$ Department of Electronics and Electrical Engineer at University College London, London, WC1E 7JE, UK \\ ${ }^{2}$ Finisar Corporation, Fremont, CA 94538 USA \\ ${ }^{3}$ School of Information and Communication Technology, Royal Institute of Technology, Kista SE-164 40, Sweden \\ e-mail address: zhixin.liu@ucl.ac.uk; yasuhiro.matsui@finisar.com
}

\begin{abstract}
We demonstrate gain-switched pulse generation at a record-high repetition rate of $50 \mathrm{GHz}$ by injection locking a cavity-enhanced DFB laser. More than $50 \mathrm{GHz}$ carrier-photon resonance is achieved by using the detuned-loading and photon-photon resonance effects.
\end{abstract}

\section{Introduction}

Gain switching of directly modulated lasers (DML) provides a compact and low cost way to generate periodic pulses for communications and clock synchronization [1,2]. Assisted with external optical injection locking (OIL), gainswitched lasers have significantly improved performance compared to solitary gain-switched lasers, including suppressed chirp, reduced jitter and linewidth, showing frequency comb generation with a free spectral range (FSR) of up to $33 \mathrm{GHz}$ for wavelength-division multiplexed (WDM) communication systems [3]. In the meantime, the recent trend on high baud rate data communications has imposed new demands on the FSR of comb sources to be $50 \mathrm{GHz}$ and above. To meet the challenge, the gain-switched OIL-DMLs need to be modulated at a minimum frequency of $50 \mathrm{GHz}$.

For a solitary semiconductor laser, the intrinsic modulation bandwidth is associated to the carrier-photon resonance frequency $\left(F_{r}\right)$, which is fundamentally limited by the laser material and the pump current density. To overcome this limit, the self-feedback approach was employed by creating a photon-photon resonance (PPR) between the external mode for distributed feedback (DFB) laser. Examples include the self-feedback laser [4] and the short-cavity membrane distributed reflector (DR) laser [4]. By harnessing the strong optical confinement effect formed by air, the short-cavity membrane DR laser showed a 3-dB bandwidth of $107 \mathrm{GHz}$ [5]. Using a standard semiconductor laser manufacturing process, Matsui et al. earlier demonstrated a short-cavity distributed reflector (DR) laser with an $F_{r}$ of $30 \mathrm{GHz}$ and a PPR response at $50 \mathrm{GHz}$ [6] by utilizing both PPR and detuned-loading effects, in which the FM-AM conversion by DBR mirror enhanced the effective differential gain. This result was supported by large signal data modulation of up to $112 \mathrm{~Gb} / \mathrm{s}$ 4-level pulse modulation format (PAM4) with an extinction ratio of $4.8 \mathrm{~dB}$. In general, PPR or detuned-loading effects are sensitive to the bias condition. However, its potential and stability under gain switching has not been studied. Importantly, to generate low jitter and chirpsuppressed comb, external OIL is needed [1]. However, the joint effects of the detuned-loading and PPR have not been well understood for gain-switched lasers under OIL.

In this paper, we investigate the gain-switching feature of a cavity-enhanced DFB laser with an external OIL at a record high frequency of $50 \mathrm{GHz}$. The cavity-enhanced DFB laser used in the experiment exploited the combined effect of the detuned-loading and PPR created by an integrated coupled-cavity section, which realized an $F_{r}$ of $47 \mathrm{GHz}$ and a corresponding 3-dB bandwidth of $52.5 \mathrm{GHz}$ under small signal measurement. By applying the OIL, the PPR and OIL-enhanced $F_{r}$ further extended the modulation bandwidth. Under a large modulation current of > $100 \mathrm{mApp}$, we generate 6-ps-width short pulses at a repetition rate of $50 \mathrm{GHz}$. The OIL suppresses the frequency chirp and results in a flat comb generation with less than $2 \mathrm{~dB}$ power fluctuation across $100 \mathrm{GHz}$ bandwidth. In addition, we show that the repetition rate (or the FSR of the comb) can be easily halved by tuning the laser bias current slightly away from the stable operation condition, which provides a convenient way to divide the clock for potential applications such as optical clock distribution [7].

\section{Experimental Setup}

Fig. 1(a) shows the experimental setup. The cavity-enhanced DFB laser used in this experiment contains a DFB section of $80 \mu \mathrm{m}$ length and an integrated passive waveguide section with a low reflectivity of $\sim 3 \%$ at the facet, namely " $\mathrm{DFB}+\mathrm{R}$ laser". This low reflection creates a strong etalon filter effect in the laser cavity, and thus generates both the PPR effect and the detuned-loading effect. Noting that, the detuning-loading effect does not exist in either the PFL [4] or the membrane DR laser [5]. In these two lasers, the facets have either a high reflectivity (HR) coating 
or a 30\% mirror reflection, respectively. With such higher reflection, the etalon filter acts as an "all-pass filter", and thus the detuned-loading effect disappears. Therefore, it is important to keep the reflectivity to $\sim 3 \%$ in order to exploit the detuned-loading effect. As a result, the BW was improved from $24 \mathrm{GHz}$ for a solitary DFB laser to $52.5 \mathrm{GHz}$ for a $\mathrm{DFB}+\mathrm{R}$ laser. In our experiment, the DC bias current and the $50-\mathrm{GHz} \mathrm{RF}$ signals were combined via a $55-\mathrm{GHz}$ bias tee and the output was supplied to the laser chip via a $65-\mathrm{GHz}$ RF probe. The $50 \mathrm{GHz}$ sinusoidal RF signal was boosted to $30 \mathrm{dBm}$ using a narrow band $(48-52 \mathrm{GHz})$ power amplifier. Due to the loss in the RF signal chain, the signals applied on the laser chip was about $27 \mathrm{dBm}$.
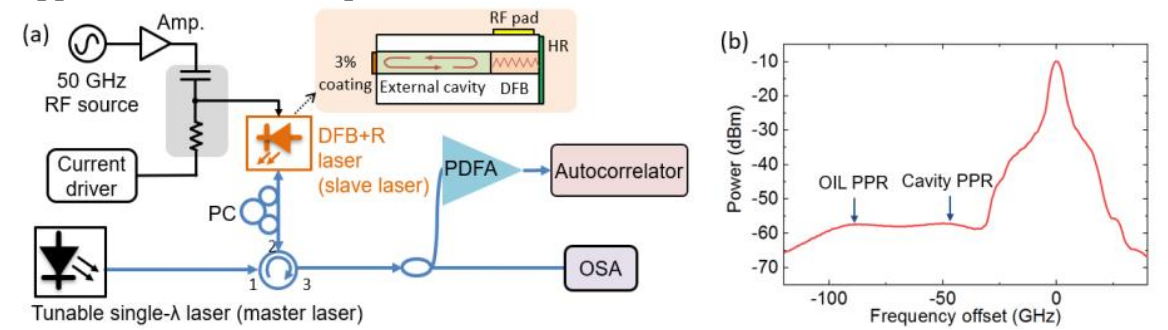

Fig.1 (a) Experimental setup; (b) Optical spectrum of the OIL-DML. OSA: Optical spectrum analyzer; Amp.: RF amplifier; PDFA: Praseodymium-Doped Fiber Amplifier; DFB: distributed feedback; PC: polarization controller.

A tunable laser emitting at the telecom O-band was employed as the master laser. The master light was launched into the slave laser via a lens system after passing through a circulator and a polarization controller (PC), which ensured maximum light coupling efficiency into the slave laser cavity. The output of the slave laser was emitted from the port 3 of the circulator and was split to two branches by a 50:50 splitter. The lower branch was sent to an optical spectrum analyzer (OSA, $0.02 \mathrm{~nm}$ resolution) and the upper branch was sent to a Praseodymium-Doped Fiber Amplifier (PDFA), which amplified the optical power optical power to $18 \mathrm{dBm}$ for an autocorrelator (Femtochrome FR-103XL) to perform the short pulse measurement.

Both the injection power and the detuning frequency affects the dynamics of the OIL-DML. In this experiment, we kept the injection power at $3 \mathrm{dBm}$ for stable performance. The frequency detuning $\left(\Delta \mathrm{f}=\mathrm{f}_{\mathrm{inj}} \mathrm{f}_{\mathrm{free}}\right)$, defined as the difference between the frequency of the master light $\left(\mathrm{f}_{\mathrm{inj}}\right)$ and the frequency of the free running slave laser ( $\left.f_{\text {free}}\right)$, was optimized for obtaining stable pulsed outputs. The externally seeded light locks the output of the slave laser to the frequency of the injected signal $\left(f_{\text {inj }}\right)$ and creates a second PPR between the injected frequency and the blue shifted DFB mode at around $85 \mathrm{GHz}$ [8]. In this paper, we call this 'OIL PPR' to differentiate from the 'cavity PPR' that arises from integrated coupled-cavities, which is located at around $50 \mathrm{GHz}$, as shown in Fig. 1 (b).

\section{Results}

Fig. 2 shows the light-current (LI) characteristics of the cavity-enhanced DFB laser under both DC drive and gain switching. To avoid the damage on the laser modulated under 27-dBm RF power without DC bias, we only measured the threshold current under $\mathrm{CW}$ condition. The $\mathrm{CW}$ laser showed a threshold current of $4.2 \mathrm{~mA}$ and a slope efficiency of 0.33 W/A. Under DC operation, two kinks around 37 and $67 \mathrm{~mA}$ due to the mode hop among compound cavity modes were observed. Under gain switching, only one kink around $55 \mathrm{~mA}$ was observed due to the higher local Joule heating in the DFB section under large RF modulation. Around this condition, the main lasing mode experiences a strong frequency-dependent cavity loss modulation. This effect, known as a detuned-loading effect, effectively enhances the differential gain and therefore can increase carrier-photon resonance frequency $F_{r}$. In the gain switching experiment, this bias condition corresponded to the shortest pulse condition, which exploits both detuned-loading and PPR effects to reduce the pulse width of the gain-switched pulses.

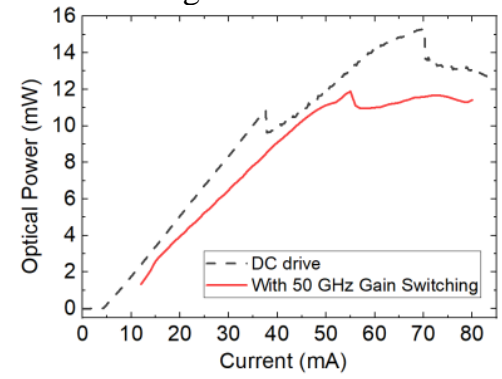

Fig. 2 Light-current measurement (LI curve) of the solitary laser under DC current drive (red solid line) and 50-GHz gain switching. (dashed line).
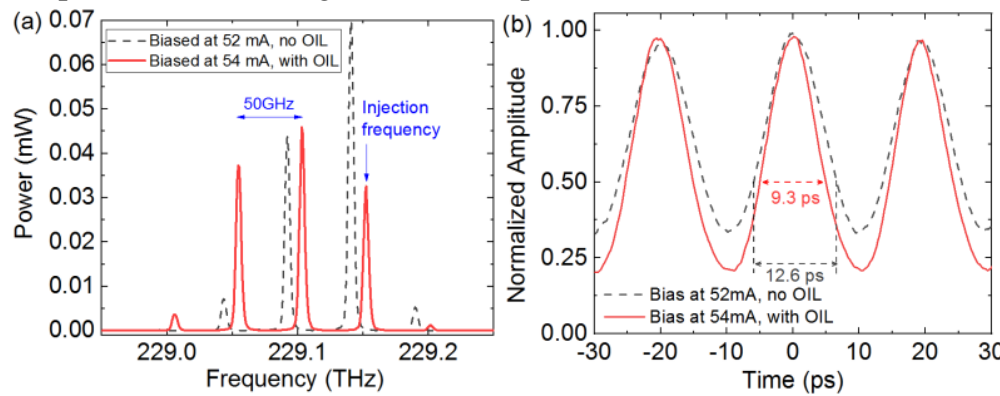

Fig. 3 Gain switching results: (a) Optical spectra without and without OIL in linear scale; (b) original autocorrelation measurements. 
The gain-switched cavity-enhanced laser was biased at $54 \mathrm{~mA}$, generating a stable single mode output with a side mode suppression ratio (SMSR) greater than $30 \mathrm{~dB}$. Fig.3 (a) shows the optical spectra of the gain-switched DML with and without OIL, in solid red line and black dashed line, respectively. The spectra are plotted in a linear scale for a direct observation of the optical bandwidth. With OIL, the gain-switched DML outputs clearly resolved $50-\mathrm{GHz}$ spacing tones with less than $2 \mathrm{~dB}$ power variation of the three center lines, making it a promising light source for flat comb generation with the use of expansion techniques [1]. The time domain performance measured using the autocorrelator is shown in Fig.3 (b). The full width half maximum (FWHM) values with and without OIL were $12.6 \mathrm{ps}$ and $9.3 \mathrm{ps}$, respectively, before deconvolution. Assuming $\mathrm{sech}^{2}$ shape, the calculated pulse widths are $8.2 \mathrm{ps}$ and $6 \mathrm{ps}$, indicating an intrinsic oscillation frequency of 38 and $52 \mathrm{GHz}$, for gain switched DML with and without OIL, respectively. Compared to previous gain-switched demonstration at $20 \mathrm{GHz}$ that showed a pulse width of $12.3 \mathrm{ps}$ with an $F_{r}$ of $25 \mathrm{GHz}$, we achieved a reduction of pulse width by a factor of 2 , and the same factor for the improvement of the oscillation frequency. Assuming linear chirp, the calculated time-bandwidth products were 0.9 and 0.66 with and without seeding, respectively, indicating the potential pulse width of $2.1 \mathrm{ps}$ and $4.0 \mathrm{ps}$ after compression, respectively.

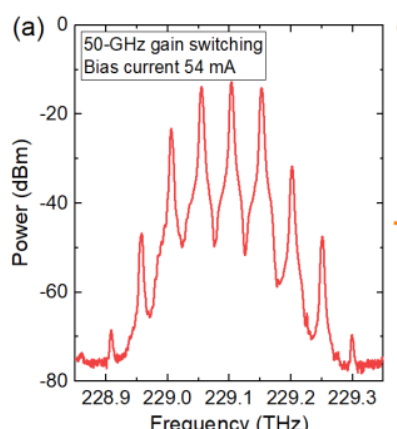

Frequency $(\mathrm{THz})$

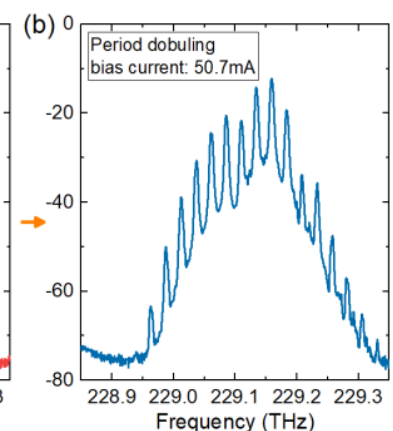

Frequency $(\mathrm{THz})$

Fig. 4 Optical spectra when the gain switched laser evolves from (a)

$50-\mathrm{GHz}$ repetition rate pulse generation to (b) period doubling.
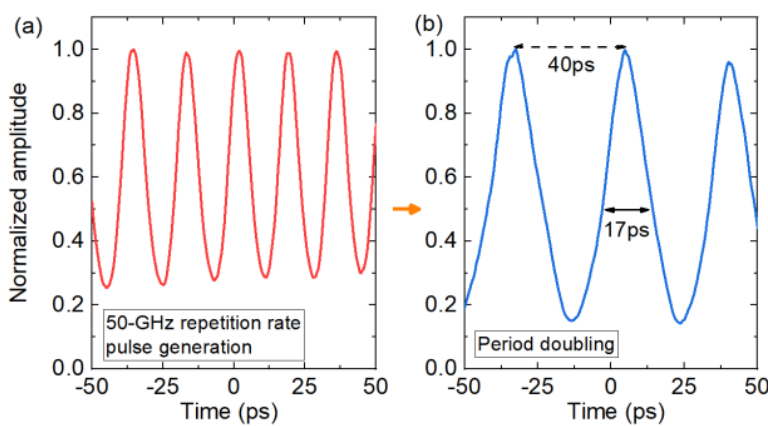

Fig. 5 Autocorrelator measurement when the gain switched laser evolves from (a) 50-GHz repetition rate pulse generation to (b) period doubling.

In addition to 50-GHz pulse generation, we also demonstrate the period doubling effect that converts the pulse repetition rate from $50 \mathrm{GHz}$ to $25 \mathrm{GHz}$ by simply tuning the bias current away to $50.7 \mathrm{~mA}$. Fig. 4 (a) and Fig.4 (b) show the optical spectra of the stable gain switching and the period doubling, both show distinguishable tones at $50 \mathrm{GHz}$ and $25 \mathrm{GHz}$ FSR, respectively. Their corresponding time autocorrelator measurement results are shown in Fig. 5 (a) and Fig.5 (b), respectively. The period doubling effect occurs when the gain switching frequency becomes higher than the carrier-photon resonance frequency when laser parameters change, which also leads to an increase of the pulse width to $11 \mathrm{ps}$ (assuming $\mathrm{Sech}^{2}$ pusle shape). The period doubling effects is conventionally of interest in the laser physics community. However, the simplicity of halving the repetition rate may benefit the optical communication community in applications such as photonic clock divider and clock dissemination.

\section{Conclusions}

We demonstrate gain switching at a record high repetition rate of $50 \mathrm{GHz}$ with a short pulse width of $6 \mathrm{ps}$. The high carrier-photon resonance was achieved by jointly exploiting the detuning loading and the PPR effects using the integrated cavity mode and an external OIL. Our results confirm that the PPR can increase the bandwidth for large signal modulation, which complements the study on small signal modulation bandwidth in existing literature. We report a flat optical spectrum with less than $2 \mathrm{~dB}$ power variation to support future research on comb generation, and report 50 to $25 \mathrm{GHz}$ period doubling for the first time.

The authors thank Femtochrome for loan of the autocorrelator. This work was supported by EPSRC grant EP/R041792/1, EP/R035342/1, and Royal Society RSGIR1\180200.

\section{References}

[1] P. M. Anandarajah et al., "Generation of coherent multicarrier signals by gain switching of discrete mode lasers," Photonics J., 3, 112-122 (2011).

[2] L.C. Comandar et al., "Quantum key distribution without detector vulnerabilities using optically seeded lasers," Nat. Photon., 10, (2016)

[3] P. M. Anandarajah et al., "Flexible Optical Comb Source for Super Channel Systems," in Proc. OFC, OTh3I.8 (2013).

[4] M. Radziunas et al., "Improving the Modulation Bandwidth in Semiconductor Lasers by Passive Feedback," JSTQE, 13, 136-142 (2007).

[5] S. Yamaoka et al., "239.3-Gbit/s Net Rate PAM-4 Transmission using Directly Modulated Membrane Lasers on High-Thermal-Conductivity $\mathrm{SiC}$, , in Proc. ECOC, PD.2.1 (2019).

[6] Y. Matsui et al., "55 GHz Bandwidth Distributed Reflector Laser,” J. Light. Technol., 35, 397-403 (2017).

[7] S. Chan and J. Liu, "Microwave frequency division and multiplication using an optically injected semiconductor laser," JQE, 41, (1998).

[8] A. Murakami et al., "Cavity resonance shift and bandwidth enhancement in semiconductor lasers with strong light injection," JQE, 39, (2003) 